\title{
Übergabe der Herausgeberschaft des JfB
}

\author{
Nikolaus Franke • Gerhard Speckbacher
}

Online publiziert: 8. April 2011

(C) Wirtschaftsuniversität Wien, Austria 2011

Im Jahr 2003 trat die Wirtschaftsuniversität Wien mit der Bitte an uns heran, das traditionsreiche, vor über einem halben Jahrhundert gegründete Journal für Betriebswirtschaft im Profil zu schärfen und als eine der führenden wissenschaftlichen Zeitschriften im deutschsprachigen Raum neu zu positionieren. In den acht Jahren ist viel passiert. Wir haben Beiräte, Positionierung, sämtliche Prozesse, den Verlagspartner und den Außenauftritt neu gefasst und mit unseren Entscheidungen viel Glück gehabt. Wir haben 256 Einreichungen über bis zu vier Reviewrunden mit hunderten von Gutachtern betreut und davon 49 State-of-the-Art Reviews schließlich publiziert. Viele von ihnen sind einflussreich und werden oft zitiert, manche sind echte „Meilensteine" geworden. Sie alle helfen mit, die oft beklagte Fragmentierung und Unübersichtlichkeit der betriebswirtschaftlichen Forschung zu reduzieren.

Von Anfang an war unsere Position, dass die Herausgeberschaft einer Zeitschrift zeitlich befristet sein sollte. Das Beispiel praktisch aller Spitzenzeitschriften zeigt, wie wichtig neben der Kontinuität auch der Wechsel ist. Wir hatten seinerzeit eine Befristung auf fünf Jahre als ideal angesehen, weil man über diesen Zeitraum eine neue Positionierung nachhaltig umsetzen kann und weil das auch für uns noch ein überschaubarer Planungshorizont war. Tatsächlich ist es gelungen, das JfB zu einer der angesehensten und meistgelesenen deutschsprachigen Zeitschriften (VHB Ranking 2008) zu entwickeln und nun sind aus den fünf Jahren eben acht Jahre geworden. Dass wir die geplante Befristung etwas aus den Augen verloren hatten, liegt an der Fülle der Aufgaben und an der großen Anerkennung seitens des Rektorats der Wirtschaftsuniversität Wien und der Beiräte für die erzielten Erfolge. Natürlich hat auch

\footnotetext{
N. Franke $(\bowtie) \cdot$ G. Speckbacher

Wirtschaftsuniversität Wien, Wien, Österreich

e-mail: nikolaus.franke@wu.ac.at

G. Speckbacher

e-mail: gerhard.speckbacher@wu-wien.ac.at
} 
eine Rolle gespielt, dass uns die Arbeit mit Autoren, Reviewern, Redaktion, Verlag und Lesern immer viel Spaß gemacht hat. Doch wie heißt es so schön? Finis coronat opus. Bereits im vergangenen Jahr haben wir daher das Rektorat der WU um Unterstützung bei der Suche nach geeigneten Nachfolgern gebeten.

Unser gemeinsames „Dream Team“ besteht aus folgenden Personen:

- Engelbert Dockner ist Professor für Finance und Corporate Strategy an der Wirtschaftsuniversität Wien. Seine Forschungsgebiete sind Investitionstheorie, Produkt- und Finanzmarktinteraktionen, Industrial Organization, Risikomanagement und Spieltheorie. Wissenschaftliche Aufsätze von Engelbert Dockner sind unter anderem in American Economic Review, Economics Letters, Journal of Economic Behavior and Organization, Journal of Economic Dynamics and Control, Journal of Economic Theory, Management Science und Marketing Science erschienen.

- Jonas Puck ist Professor für International Business an der Wirtschaftsuniversität Wien. Seine Forschungsgebiete sind International Human Resource Management, Cross-Cultural Management, Emerging Markets und International M\&A. Wissenschaftliche Aufsätze von Jonas Puck sind unter anderem in Journal of International Business Studies, Journal of International Management, Long Range Planning, International Business Review und International Journal of Human Resource Management erschienen.

- Thomas Reutterer ist ao. Professor für Marketing an der Wirtschaftsuniversität Wien. Seine Forschungsgebiete sind Customer Relationship Management und Direkt-Marketing, die Analyse dynamischer Kunden-Firmen-Beziehungen sowie die Auswirkungen von Entscheidungshilfen auf das Entscheidungsverhalten im Marketing-Management. Wissenschaftliche Aufsätze von Thomas Reutterer sind unter anderem in Journal of Marketing, Marketing Science, European Journal of Operational Research, Computers \& Operations Research, Journal of Retailing and Consumer Services und Industrial Marketing Management erschienen.

Wir haben damit hinsichtlich der thematischen Breite, der Forschungskompetenz und der internationalen Reputation ideale künftige Herausgeber des JfB gefunden. Das neue Herausgeberteam ist engagiert und unternehmerisch und hat bereits eine Vielzahl von Ideen zur künftigen Weiterentwicklung des JfB mit uns diskutiert. Wir sind sicher, dass das neue Herausgeberteam das JfB sehr erfolgreich weiterentwickeln wird. 